\title{
AMENABILITY AND TOPOLOGICAL CENTRES OF THE SECOND DUALS OF BANACH ALGEBRAS
}

\author{
F. Ghahramani and J. LaAli
}

\begin{abstract}
Let $\mathfrak{A}$ be a Banach algebra and let $\mathfrak{A}^{\star \star}$ be the second dual algebra of $\mathfrak{A}$ endowed with the first or the second Arens product. We investigate relations between amenability of $\mathfrak{A}^{\star \star}$ and Arens regularity of $\mathfrak{A}$ and the rôle of topological centres in amenability of $\mathfrak{A}^{\star \star}$. We also find conditions under which weak amenability of $\mathfrak{A}^{\star \star}$ implies weak amenability of $\mathfrak{A}$.
\end{abstract}

\section{INTRODUCTION AND PRELIMINARIES}

Let $\mathfrak{A}$ be a Banach algebra and $\mathfrak{A}^{\star \star}$ be the second dual space of $\mathfrak{A}$ endowed with the first or the second Arens product. In [2] the first author, Loy and Willis studied some implications of amenability and weak amenability of $\mathfrak{A}^{\star \star}$; special emphasis was put on the case when $\mathfrak{A}$ was a Banach algebra related to a locally compact group. These studies have lead to the work done in $[\mathbf{1}, \mathbf{4}, \mathbf{7}, \mathbf{8}, \mathbf{9}]$. In this paper we consider the following two questions

1. Is $\mathfrak{A}$ Arens regular when $\mathfrak{A}^{\star \star}$ is amenable?

2. Is $\mathfrak{A}$ weakly amenable when $\mathfrak{A}^{\star \star}$ is weakly amenable?

For the origin of these questions see $[2,3]$. We show that under certain additional assumptions on $\mathfrak{A}$ or $\mathfrak{A}^{\star \star}$ the answer to either one of these questions is positive. We also explore the rôle of the topological centres in amenability of $\mathfrak{A}^{\star \star}$.

Throughout this paper, the first (second) Arens product is denoted by $\square$ (respectively $\diamond)$. These products can be defined by

$$
F \square G=\text { weak }^{\star} \lim _{i} \lim _{j} \widehat{f}_{i} \widehat{g}_{j} \quad \text { and } F \diamond G=\text { weak }^{\star} \lim _{i} \lim _{j} \widehat{f}_{i} \widehat{g}_{j},
$$

where $\left(f_{i}\right)$ and $\left(g_{j}\right)$ are nets of elements of $\mathfrak{A}$ such that $\widehat{f}_{i} \rightarrow F$ and $\widehat{g}_{i} \rightarrow G$, in the weak topology. The first topological centre of $\mathfrak{A}^{\star \star}$ is

$$
\begin{aligned}
Z_{1} & =\left\{y \in \mathfrak{A}^{\star \star}: x \mapsto y \square x \text { is weak* continuous }\right\} \\
& =\left\{y \in \mathfrak{A}^{\star \star}: y \square x=y \diamond x, \text { for all } x \in \mathfrak{A}^{\star \star}\right\},
\end{aligned}
$$

Received 26th April, 2001.

The first author's research was supported by NSERC grant 36640-98.

Copyright Clearance Centre, Inc. Serial-fee code: 0004-9727/02 \$A2.00+0.00. 
and the second topological centre is defined by

$$
Z_{2}=\left\{y \in \mathfrak{A}^{\star \star}: x \mapsto x \diamond y \text { is weak* continuous }\right\} \text {. }
$$

We note that $\mathfrak{A}$ is Arens regular if and only if $Z_{1}=\mathfrak{A}^{\star \star}$, or $Z_{2}=\mathfrak{A}^{\star \star}$. See [2] and [7] for properties of Arens products and topological centres.

A Banach algebra $\mathfrak{A}$ is amenable if every continuous derivation $D: \mathfrak{A} \rightarrow X^{\star}$ is inner, for every Banach $\mathfrak{A}$-bimodule $X$. If all the continuous derivations from $\mathfrak{A}$ into $\mathfrak{A}^{\star}$ (the special case of $X=\mathfrak{A}$ ) are inner, then $\mathfrak{A}$ is weakly amenable. There are alternative formulations of the notion of amenability, of which we need the following two, first introduced in [6]. The Banach algebra $\mathfrak{A}$ is amenable if and only if either, and hence both, of the following hold,

(i) $\mathfrak{A}$ has an approximate diagonal, that is a bounded net $\left(m_{i}\right) \subset(\mathfrak{A} \widehat{\otimes} \mathfrak{A})^{\star \star}$ such that for each $x \in \mathfrak{A}, x \cdot m_{i}-m_{i} \cdot x \rightarrow 0, \pi\left(m_{i}\right) x \rightarrow x$;

(ii) $\mathfrak{A}$ has a virtual diagonal, that is an element $M \in(\mathfrak{A} \widehat{\otimes} \mathfrak{A})^{\star \star}$ such that for each $x \in \mathfrak{A}, x \cdot M=M \cdot x$, and $\left(\pi^{\star \star} M\right) \cdot x=\widehat{x}$; here $\pi: \mathfrak{A} \widehat{\otimes} \mathfrak{A} \rightarrow \mathfrak{A}$ is specified by $\pi(a \otimes b)=a b(a, b \in \mathfrak{A})$.

\section{AmENABILITY of CERTAIN SUbALgEBRAS of $\mathfrak{A}^{\star \star}$}

In [2, Theorem 1.8] it was shown that if $\mathfrak{A}^{\star \star}$ is amenable (with either one of the Arens products), then $\mathfrak{A}$ is amenable. In the following proposition we strengthen the above cited result. We have assumed that $\mathfrak{A}^{\star \star}$ has first Arens product. The image of $\mathfrak{A}$ in $\mathfrak{A}^{\star \star}$ under the canonical mapping is denoted by $\widehat{\mathfrak{A}}$.

Proposition 1.1. Let $B$ be a closed subalgebra of $\mathfrak{A}^{\star \star}$ such that $\widehat{\mathfrak{A}} \subseteq B$. If $B$ is amenable, then $\mathfrak{A}$ is amenable.

Proof: By [2, Lemma 1.7] there is a continuous linear mapping $\psi: \mathfrak{A}^{\star \star} \widehat{\otimes} \mathfrak{A}^{\star \star} \rightarrow$ $(\mathfrak{A} \widehat{\otimes} \mathfrak{A})^{\star \star}$ such that for $a, b, x \in \mathfrak{A}$ and $m \in \mathfrak{A}^{\star \star} \widehat{\otimes} \mathfrak{A}^{\star \star}$ the following hold.
(i) $\psi(\widehat{a} \otimes \widehat{b})=(a \otimes b)^{\wedge}$;
(ii) $\psi(m) \cdot x=\psi(m \cdot x)$;
(iii) $x \cdot \psi(m)=\psi(x \cdot m)$;
(iv) $\left(\pi_{\mathfrak{A}}\right)^{\star \star}(\psi(m))=\pi_{\mathfrak{A} * *}(m)$.

From the definition of projective tensor norm we see that when both $B \widehat{\otimes} B$ and $\mathfrak{A}^{\star \star} \widehat{\otimes} \mathfrak{A}^{\star \star}$ are equipped with the projective tensor norm, then the mapping $J: B \widehat{\otimes} B \rightarrow \mathfrak{A}^{\star \star} \widehat{\otimes} \mathfrak{A}^{\star \star}$ specified by $J\left(b_{1} \otimes b_{2}\right)=b_{1} \otimes b_{2},\left(b_{1}, b_{2} \in B\right)$ is norm decreasing. Let $\left(m_{i}\right)$ be an approximate diagonal for $B$, and set $\Phi=\psi \circ J$. Then for all $x \in \mathfrak{A}, \Phi\left(m_{i}\right) \cdot x-x \cdot \Phi\left(m_{i}\right) \rightarrow 0$ and $\pi_{\mathfrak{A}}^{\star \star}\left(\Phi\left(m_{i}\right)\right) \cdot x \rightarrow x$. If $M$ is a weak ${ }^{\star}$-cluster point of $\left(\Phi\left(m_{i}\right)\right)$ in $(\mathfrak{A} \widehat{\otimes} \mathfrak{A})^{\star \star}$, then, for each $x \in \mathfrak{A}, M \cdot x=x \cdot M$ and $\pi_{\mathfrak{a}}^{\star \star}(M) \cdot x=x$, and so $M$ is a virtual diagonal for $\mathfrak{A}$.

Corollary 1.2. Suppose that $Z_{1}$ (or $Z_{2}$ ) is amenable. Then $\mathfrak{A}$ is amenable. 
For a Banach algebra $\mathfrak{A}$, let $\mathfrak{A}^{\boldsymbol{p} p}$ be the Banach algebra with underlying Banach space same as $\mathfrak{A}$ and with product $\circ$ given by $a \circ b=b a$.

Proposition 1.3. Let $\mathfrak{A}$ be a Banach algebra. Then

(i) $\mathfrak{A}$ is amenable if and only if $\mathfrak{A}^{o p}$ is amenable.

(ii) Let $\mathfrak{A}$ be commutative. Then $\left(\mathfrak{A}^{\star \star}, \square\right)$ is amenable if and only if $\left(\mathfrak{A}^{\star \star}, \diamond\right)$ is amenable.

ProOF:

(i) This is trivial.

(ii) Take $F, G \in \mathfrak{A}^{\star \star}$, and let $\left(f_{i}\right),\left(g_{j}\right)$ be nets in $\mathfrak{A}$ such that $w^{\star}-\lim _{\boldsymbol{i}} \widehat{f}_{i}=F$ and $w^{\star}-\lim _{j} \widehat{g}_{j}=G$. Then

$$
F \square G=w^{\star}-\lim _{i} w^{\star}-\lim _{j} \widehat{f}_{i} \widehat{g}_{j}=w^{\star}-\lim _{i} w^{\star}-\lim _{j} \widehat{g}_{j} \circ \widehat{f}_{i}=G \diamond F,
$$

and so $\left(\mathfrak{A}^{\star \star}, \square\right)=\left(\mathfrak{A}^{\star \star}, \diamond\right)^{o p}$. By $(\mathrm{i}),\left(\mathfrak{A}^{\star \star}, \square\right)$ is amenable if and only if $\left(\mathfrak{A}^{\star \star}, \diamond\right)$ is amenable.

Proposition 1.4. Let $\mathfrak{A}$ be a Banach algebra with a continuous anti-isomorphism $\lambda: \mathfrak{A} \rightarrow \mathfrak{A}$. Then, $\left(\mathfrak{A}^{\star \star}, \square\right)$ is amenable if and only if $\left(\mathfrak{A}^{\star \star}, \diamond\right)$ is amenable. A similar conclusion holds if $\lambda$ is a continuous involution.

ProOf: Let $\lambda: \mathfrak{A} \rightarrow \mathfrak{A}$ be a continuous anti-isomorphism. Set $\left(\mathfrak{A}^{\star \star}, \square\right)=A$ and $\left(\mathfrak{A}^{\star \star}, \diamond\right)=B$. Take $F, G$ in $A$ and let $\left(f_{i}\right)$ and $\left(g_{j}\right)$ be nets in $\mathfrak{A}$ such that weak ${ }^{\star}-\lim _{i} \widehat{f}_{i}=$ $F$, weak $^{\star}-\lim _{j} \hat{g}_{j}=G$.

Let $\lambda^{\star}: A \rightarrow B$, be the second adjoint of $\lambda$. Then

$$
\begin{aligned}
\lambda^{\star \star}(F \square G) & =w^{\star}-\lim _{i} w^{\star}-\lim _{j} \lambda^{\star \star}\left(\hat{f}_{i} \widehat{g}_{j}\right) \\
& =w^{\star}-\lim _{i} w^{\star}-\lim _{j}\left(\lambda\left(f_{i}\right) \lambda\left(g_{j}\right)\right)^{\wedge} \\
& =w^{\star}-\lim _{i} w^{\star}-\lim _{j} \lambda^{\star \star}\left(\widehat{g}_{j}\right) \lambda^{\star \star}\left(\widehat{f}_{i}\right) \\
& =\lambda^{\star \star}(G) \diamond \lambda^{\star \star}(F) .
\end{aligned}
$$

Hence $\lambda^{\star \star}$ is an isomorphism from $A$ onto $B^{o p}$ and so by Proposition 1.3 (i) $B$ is amenable.

The proof in the case when $\lambda$ is an involution follows similar lines.

Recall that $\mathfrak{A}^{\star}$ is said to factor on the left if $\mathfrak{A}^{\star} \cdot \mathfrak{A}=\mathfrak{A}^{\star},[10]$. When $\mathfrak{A}$ has a bounded approximate identity and $\mathfrak{A}^{\star \star}$ has an identity, $\mathfrak{A}^{\star}$ factors on the left $[\mathbf{1 0}]$.

THEOREM 1.5. Suppose that $\left(\mathfrak{A}^{\star \star}, \square\right)$ is amenable and $\widehat{\mathfrak{A}} \square \mathfrak{A}^{\star} \subset Z_{1}$. Then $\mathfrak{A}$ is Arens regular. 
Proof: Since $\left(\mathfrak{A}^{\star \star}, \square\right)$ is amenable it has an identity [2]. Also, amenability of $\mathfrak{A}^{\star \star}$ necessitates amenability of $\mathfrak{A},([2])$, and so $\mathfrak{A}$ has a bounded approximate identity. Hence $\mathfrak{A}^{\star}$ factors on the left; $\mathfrak{A}^{\star} \cdot \mathfrak{A}=\mathfrak{A}^{\star}$. Let $f \in \mathfrak{A}^{\star}$. Then $f=g \cdot a$, for some $g \in \mathfrak{A}^{\star}$ and $a \in \mathfrak{A}$. Let $m, n \in \mathfrak{A}^{\star \star}$, and $f \in \mathfrak{A}^{\star}$. Then, since $\widehat{a} \square m \in Z_{1}$ and $\widehat{a} \square m=\widehat{a} \diamond m$, we have

$$
\begin{aligned}
\langle m \square n, f\rangle & =\langle m \square n, g \cdot a\rangle \\
& =\langle\widehat{a} \square(m \square n), g\rangle=\langle(\widehat{a} \square m) \square n, g\rangle \\
& =\langle(\widehat{a} \square m) \diamond n, g\rangle=\langle(\widehat{a} \diamond m) \diamond n, g\rangle \\
& =\langle\widehat{a} \diamond(m \diamond n), g\rangle=\langle m \diamond n, g \cdot a\rangle \\
& =\langle m \diamond n, f\rangle,
\end{aligned}
$$

and so $m \square n=m \diamond n$, showing that $\mathfrak{A}$ is Arens regular.

\section{WEAK AMENABILITY OF $\mathfrak{A}^{\star \star}$}

Let $\mathfrak{A}^{2}=\operatorname{span}\{a b: a, b \in \mathfrak{A}\}$.

It is known that if the Banach algebra $\mathfrak{A}$ is weakly amenable, then $\mathfrak{A}^{2}$ is dense in $\mathfrak{A}$. The following is a positive result in the direction of answering the question of whether weak amenability of $\mathfrak{A}^{\star \star}$ implies weak amenability of $\mathfrak{A}$.

Proposition 2.1. Suppose that $\mathfrak{A}^{\star \star}$ is weakly amenable. Then $\mathfrak{A}^{2}$ is dense in $\mathfrak{A}$.

Proof: Let $a \in \mathfrak{A}$. Since $\mathfrak{A}^{\star \star}$ is weakly amenable $\mathfrak{A}^{\star \star}$ is equal to the closure of $\left(\mathfrak{A}^{\star \star}\right)^{2}$. Hence there exists a sequence $\left(s_{n}\right) \subset\left(\mathfrak{A}^{\star \star}\right)^{2}$ such that $s_{n}=\sum_{k=1}^{K(n)} M_{n, k} \square N_{n, k}$ and
norm- $\lim s_{n}=\widehat{a}$.

On the other hand, for each $n$ and $k$, there exist nets $\left\{a_{n, k, i}: i \in I\right\}$ and $\left\{b_{n, k, j}: j \in\right.$ $J\}$ such that weak $-\lim _{i} \widehat{a}_{n, k, i}=M_{n, k}$ and weak $\lim _{j} \widehat{b}_{n, k, j}=N_{n, k}$. Hence

$$
M_{n, k} \square N_{n, k}=w^{\star}-\lim _{i} w^{\star}-\lim _{j} \widehat{a}_{n, k, i} \square \widehat{b}_{n, k, j}
$$

and so

$$
\widehat{a}=\operatorname{norm}-\lim _{n} w^{\star}-\lim _{i} w^{\star}-\lim _{j} \widehat{a}_{n, k, i} \square \widehat{b}_{n, k, j} .
$$

This shows that $\hat{a}$ belongs to the weak ${ }^{*}$ closure of the set $\widehat{\mathfrak{A}} \square \widehat{\mathfrak{A}}$; this means that $a$ belongs to the weak closure of $\mathfrak{A} \mathfrak{A}$. Hence $a$ is in the weak closure of $\operatorname{span}(\mathfrak{A} \mathfrak{A})$. Since $\operatorname{span}(\mathfrak{A} \mathfrak{A})$ is convex, it follows that $a$ belongs to the norm-closure of $\operatorname{span}(\mathfrak{A} \mathfrak{A})$.

Recall that a Banach algebra $\mathfrak{A}$ is a dual Banach algebra if $\mathfrak{A}=X^{\star}$ for some Banach space $X$ and $\widehat{X}$ is a submodule of the dual $\mathfrak{A}$-bimodule $\mathfrak{A}^{\star}$.

TheOREM 2.2. Suppose that $\mathfrak{A}$ is a dual Banach algebra. If $\mathfrak{A}^{\star \star}$ is weakly amenable then $\mathfrak{A}$ is weakly amenable. 
Proof: Let $\mathfrak{A}=B^{\star}$, for some Banach space $B$, such that $\widehat{B}$ is a submodule of the dual module $\mathfrak{A}^{\star}$. Let $i: B \rightarrow \mathfrak{A}^{\star}$ be the canonical mapping and let $i^{\star}$ be the adjoint of $i$. First we show that $i^{\star}$ is a homomorphism from $\left(\mathfrak{A}^{\star \star}, \square\right)$ onto $\mathfrak{A}$. If $a \in \mathfrak{A}$, then for $b \in B$, we have

$$
\left\langle i^{\star}(\widehat{a}), b\right\rangle=\langle\widehat{a}, i(b)\rangle=\langle a, b\rangle .
$$

Hence $i^{\star}(\widehat{a})=a$. Now for $F, G \in \mathfrak{A}^{\star \star}$, take two nets $\left(f_{\alpha}\right),\left(g_{\beta}\right)$ of $\mathfrak{A}$ such that $F=$ weak $^{\star}-\lim _{\alpha} \widehat{f}_{\alpha}, G=$ weak $^{\star}-\lim _{\beta} \widehat{g}_{\beta}$. Then

$$
\begin{aligned}
i^{\star}(F \square G) & =i^{\star}\left(w^{\star}-\lim _{\alpha} w^{\star}-\lim _{\beta} \widehat{f}_{\alpha} \widehat{g}_{\beta}\right)=w^{\star}-\lim _{\alpha} w^{\star}-\lim _{\beta} i^{\star}\left(\left(f_{\alpha} g_{\beta}\right)\right) \\
& =w^{\star}-\lim _{\alpha} w^{\star}-\lim _{\beta}\left(f_{\alpha} g_{\beta}\right)=w^{\star}-\lim _{\alpha} f_{\alpha} w^{\star}-\lim _{\beta} g_{\beta} \\
& \left.=w^{\star}-\lim _{\alpha}\left(i^{\star}\left(f_{\alpha}\right) w^{\star}-\lim _{\beta} i^{\star}\left(g_{\beta}\right)\right)=w^{\star}-\lim _{\alpha}\left(f_{\alpha}\right) i^{\star}(G)\right) \\
& =i^{\star}(F) i^{\star}(G) .
\end{aligned}
$$

Hence $i^{\star}$ is an algebra homomorphism from $\mathfrak{A}^{\star \star}$ onto $\mathfrak{A}$.

Now let $D: \mathfrak{A} \rightarrow \mathfrak{A}^{\star}$ be a derivation. Then $\bar{D}=i^{\star \star} \circ D \circ i^{\star}: \mathfrak{A}^{\star \star} \rightarrow \mathfrak{A}^{\star \star}$ is a derivation. In fact, let $m, n, p \in \mathfrak{A}^{\star \star}$. Then

$$
\begin{aligned}
\langle\bar{D}(m \square n), p\rangle & =\left\langle\left(i^{\star \star} \circ D \circ i^{\star}\right)(m \square n), p\right\rangle \\
& =\left\langle D\left(i^{\star}(m) i^{\star}(n)\right), i^{\star}(p)\right\rangle \\
& =\left\langle D\left(i^{\star}(m)\right) i^{\star}(n)+i^{\star}(m) D\left(i^{\star}(n)\right), i^{\star}(p)\right\rangle \\
& =\left\langle D\left(i^{\star}(m)\right), i^{\star}(n) i^{\star}(p)\right\rangle+\left\langle D\left(i^{\star}(n)\right), i^{\star}(p) i^{\star}(m)\right\rangle \\
& =\left\langle D\left(i^{\star}(m)\right), i^{\star}(n \square p)\right\rangle+\left\langle D\left(i^{\star}(n)\right), i^{\star}(p \square m)\right\rangle \\
& =\left\langle i^{\star \star}\left(D\left(i^{\star}(m)\right)\right) n \square p\right\rangle+\left\langle i^{\star \star}\left(D\left(i^{\star}(n)\right)\right), p \square m\right\rangle \\
& =\left\langle\left(i^{\star \star} \circ D \circ i^{\star}\right)(m) \cdot n+m \cdot i^{\star \star} D\left(i^{\star}(n)\right), p\right\rangle \\
& =\langle\bar{D}(m) \cdot n+m \cdot \bar{D}(n), p\rangle .
\end{aligned}
$$

Hence $\bar{D}$ is a derivation, and so from the assumption of weak amenability of $\mathfrak{A}^{\star \star}$, there exists $F \in \mathfrak{A}^{\star \star \star \star}$ such that

$$
\bar{D}(m)=m \cdot F-F \cdot m \quad\left(m \in \mathfrak{A}^{\star \star}\right) .
$$

Now $\mathfrak{A}^{\star \star}$ is naturally an $\mathfrak{A}$-bimodule and the canonical mapping $j: \mathfrak{A} \rightarrow \mathfrak{A}^{\star \star}$ is an $\mathfrak{A}$ bimodule morphism, and hence so is $j^{\star}: \mathfrak{A}^{\star \star} \rightarrow \mathfrak{A}^{\star}$. Set $f=j^{\star}(F)$. Then if $a, b \in \mathfrak{A}$, we 
have

$$
\begin{aligned}
\langle D(a), b\rangle & =\left\langle D\left(i^{\star}(\widehat{a})\right), i^{\star}(\widehat{b})\right\rangle \\
& =\left\langle i^{\star \star} D\left(i^{\star}(\widehat{a})\right), \widehat{b}\right\rangle \\
& =\langle\bar{D}(\hat{a}), j(b)\rangle \\
& =\langle\widehat{a} \cdot F-F \cdot \widehat{a}, j(b)\rangle \\
& =\left\langle j^{\star}(\widehat{a} \cdot F-F \cdot \widehat{a}), b\right\rangle \\
& =\left\langle a \cdot j^{\star}(F)-j^{\star}(F) \cdot a, b\right\rangle .
\end{aligned}
$$

Hence $D(a)=a \cdot f-f \cdot a$, and $\mathfrak{A}$ is weakly amenable.

In the proof of the next theorem we adopt the following notation. Let $\mathfrak{A}^{\star \star}$ have the first Arens product $\square$. Then for $F \in \mathfrak{A}^{\star \star \star}=\left(\mathfrak{A}^{\star \star}\right)^{\star}$ and $m \in \mathfrak{A}^{\star \star}, m \square F$ is the element of $\mathfrak{A}^{\star \star \star}$ specified by $\langle m \square F, n\rangle=\langle F, n \square m\rangle\left(m \in \mathfrak{A}^{\star \star}\right) . F \square m, m \diamond F$ and $F \diamond m$, follow similar convention and should be clear from the context.

THEOREM 2.3. Let $\mathfrak{A}$ be a Banach algebra admitting a continuous anti-homomorphism $\varphi$ such that $\varphi^{2}=1_{\mathfrak{A}}$. Then $\left(\mathfrak{A}^{\star \star}, \square\right)$ is weakly amenable if and only if $\left(\mathfrak{A}^{\star \star}, \diamond\right)$ is weakly amenable.

ProOF: Let $\varphi^{\star \star}: \mathfrak{A}^{\star \star} \rightarrow \mathfrak{A}^{\star \star}$ be the second adjoint of $\varphi$. For clarity, we introduce the following notation. $A=\left(\mathfrak{A}^{\star \star}, \square\right), A^{o p}=\left(\mathfrak{A}^{\star \star}, \bar{\square}\right), B=\left(\mathfrak{Q}^{\star \star}, \diamond\right), B^{o p}=\left(\mathfrak{A}^{\star \star}, \bar{\oslash}\right)$, so that if $F, G \in \mathfrak{A}^{\star \star}$, then $F \bar{\square} G=G \square F$ and $F \bar{\diamond} G=G \diamond F$. Using weak limits we have $\left(\varphi^{\star \star}\right)^{2}=1_{\mathfrak{A}^{\star \star}}$. Let $F, G \in \mathfrak{A}^{\star \star}$. Then $\varphi^{\star \star}(F \square G)=\varphi^{\star \star}(F) \bar{\nabla} \varphi^{\star \star}(G)$. In fact let $\left(f_{i}\right)$ and $\left(g_{j}\right)$ be nets in $\mathfrak{A}$, such that $w^{\star}-\lim _{i} \widehat{f}_{i}=F, w^{\star}-\lim _{j} \widehat{g}_{j}=G$. Then

$$
\begin{aligned}
\varphi^{\star \star}(F \square G) & =w^{\star}-\lim _{i} w^{\star}-\lim _{j} \varphi^{\star \star}\left(\widehat{f}_{i} \widehat{g}_{j}\right)=w^{\star}-\lim _{i} w^{\star}-\lim _{j}\left[\varphi\left(f_{i} g_{j}\right)\right]^{\wedge} \\
& =w^{\star}-\lim _{i} w^{\star}-\lim _{j} \varphi\left(g_{j}\right)^{\wedge} \varphi\left(f_{i}\right)^{\wedge}=\varphi^{\star \star}(G) \diamond \varphi^{\star \star}(F) \\
& =\varphi^{\star \star}(F) \bar{\diamond} \varphi^{\star \star}(G) .
\end{aligned}
$$

Similarly,

$$
\varphi^{\star \star}(F \diamond G)=\varphi^{\star \star}(F) \bar{\square} \varphi^{\star \star}(G) \quad\left(F, G \in \mathfrak{A}^{\star \star}\right) .
$$

Now suppose that $A=\left(\mathfrak{A}^{\star \star}, \square\right)$ is weakly amenable. Then $A^{o p}$ is weakly amenable. Let $D$ be a derivation from $B$ into $B^{\star}$. Then $\bar{D}=\varphi^{\star \star \star} \circ D \circ \varphi^{\star \star}$ is a derivation from $A^{o p}$ into $\left(A^{o p}\right)^{\star}$. In fact, for $m, n \in A^{o p}$, we have

$$
\begin{aligned}
\left(\varphi^{\star \star \star} \circ D \circ \varphi^{\star \star}\right)(m \bar{\square} n) & =\varphi^{\star \star \star}\left[D\left(\varphi^{\star \star}(m) \diamond \varphi^{\star \star}(n)\right)\right] \\
& =\varphi^{\star \star \star}\left[D\left(\varphi^{\star \star}(m)\right) \diamond \varphi^{\star \star}(n)+\varphi^{\star \star}(m) \diamond D\left(\varphi^{\star \star}(n)\right)\right] \\
& =\varphi^{\star \star \star}\left[D\left(\varphi^{\star \star}(m)\right] \bar{\square} n+m \bar{\square} \varphi^{\star \star \star} D\left(\varphi^{\star \star}(n)\right) .\right.
\end{aligned}
$$


Hence there exists an element $\psi \in \mathfrak{A}^{\star \star \star}$ such that for every $F \in \mathfrak{A}^{* *}$

$$
\left(\varphi^{\star \star \star} \circ D \circ \varphi^{\star \star}\right)(F)=F \overline{\bar{\sigma}} \psi-\psi \overline{\bar{\square}} F .
$$

Applying $\varphi^{\star \star}$ to the two sides of the above equation and using $\left(\varphi^{\star \star \star}\right)^{2}=1_{\mathfrak{a}^{\star \star \star}}$, we obtain

$$
D\left(\varphi^{\star \star}(F)\right)=\varphi^{\star \star}(F) \diamond \varphi^{\star \star \star}(\psi)-\varphi^{\star \star}(\psi) \diamond \varphi^{\star \star}(F) .
$$

Since $\varphi^{\star \star}$ is surjective, $D$ is inner.

\section{REFERENCES}

[1] H.G. Dales, A. Rodriguez-Palacios and M.V. Velasco, 'The second transpose of a derivation', (preprint 2000).

[2] F. Ghahramani, R.J. Loy and G.A. Willis, 'Amenability and weak amenability of second conjugate Banach algebras', Proc. Amer. Math. Soc. 129 (1996), 1489-1497.

[3] F. Gourdeau, 'Amenability of the second dual of a Banach algebra', Studia Math. 125 (1997), 75-81.

[4] E.E. Granirer, 'Amenability and semisimplicity for second duals of quotients of the Fourier algebra', J. Austral. Math. Soc. Ser. A 63 (1997), 289-296.

[5] B.E. Johnson, 'Cohomology in Banach algebras', Mem. Amer. Math. Soc. 127 (1972).

[6] B.E. Johnson, 'Approximate diagonals and cohomology of certain annihilator Banach algebras', Amer. J. Math. 94 (1972), 685-698.

[7] A.T. Lau and R.J. Loy, 'Amenability of convolution algebras', Math. Scand. 79 (1996), 283-296.

[8] A.T. Lau, R.J. Loy and G.A. Willis, 'Amenability of Banach and $C^{\star}$-algebras on locally compact groups', Studia Math. 119 (1996), 161-178.

[9] A.T. Lau and R.J. Loy, 'Weak amenability of Banach algebras on locally compact groups', J. Funct. Anal. 145 (1997), 175-204.

[10] A.T. Lau and A. Ülger, 'Topological centers of certain dual algebras', Trans. Amer. Math. Soc. 348 (1996), 1191-1212.

Department of Mathematics

University of Manitoba

Winnipeg R3T 2N2

Canada

e-mail: fereidou@cc.umanitoba.ca
Department of Mathematics

Teacher Training University

49 Mofateh Avenue

Tehran

Iran 